International Journal of Structural Stability and Dynamics

(C) World Scientific Publishing Company

\title{
OUTPUT-ONLY STRUCTURAL HEALTH MONITORING FOR DEEPWATER RISERS: EXPERIMENTAL STUDY OF WAVELET MODIFIED SOBI AND DISTRIBUTED FORCE INDEX ALGORITHM
}

\author{
CHAOJUN HUANG \\ Department of Civil $\&$ Environmental Engineering, Rice University, 6100 Main Street \\ Houston, Texas 77005, USA \\ huangchaojun00@gmail.com \\ SATISH NAGARAJAIAH \\ Department of Civil \& Environmental Engineering and Department of Mechanical Engineering \\ \& Material Science \\ Rice University, 6100 Main Street, Houston, Texas 77005, USA \\ Satish.Nagarajaiah@rice.edu \\ Received (Day Month Year) \\ Accepted (Day Month Year)
}

\begin{abstract}
Risers are the conduits between the subsea wellhead and the drilling/production platform for development, production, gas lift or water injection purposes, which are also one of the most important and the most vulnerable components for deepwater floating platforms. To address the lack of appropriate global structural health monitoring system for deepwater risers, this paper proposes a time-frequency domain approach using a wavelet modified second order blind identification (WMSOBI) method and combined distributed force change (CDFC) index. WMSOBI provides a reliable time-frequency domain identified modal properties of riser systems, even with large damping and under-determinate conditions. In addition, CDFC index generated from modal properties extracted by WMSOBI can accurately identify the damage location and damage level for both single and multiple crack scenarios. Details of experiments conducted on suspended pipe are presented. Both numerical and experimental verification are presented to validate the effectiveness of the proposed WMSOBI/CFDC algorithm and structural health monitoring system.
\end{abstract}

Keywords: output-only modal identification; structural health monitoring; blind source separation; second order blind identification; deepwater risers

\section{Introduction}

\subsection{Background}

Risers are the most important component of offshore oil platforms. Risers are the conduits between the subsea wellhead and the drilling or production platform for development, production, gas lift or water injection purposes. They can be either rigid or flexible. Risers can further be classified as top tensioned risers (TTR), steel catenary risers, and flexible risers as shown in Figure 1. 
2 Huang and Nagarajaiah

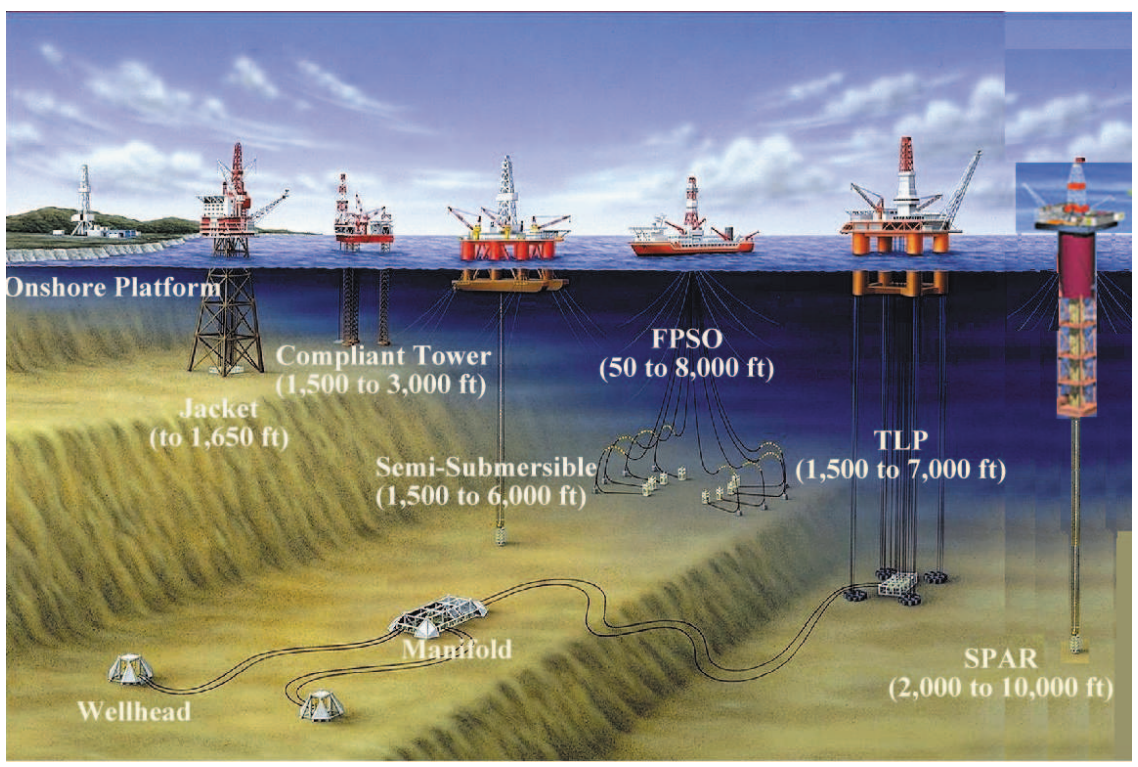

Fig. 1. Different Types of Platforms and Risers (modified from acrigs.com)

If everything on the deepwater platform functions smoothly, the crude oil and natural gas would find a safe route through risers to the global markets from the seabed wells. However, mistakes, no matter how small, can be costly, and especially the riser failure has serious consequences. Any failure incident in riser would not only be an economical and environmental disaster, but would have far reaching consequences affecting the entire community. During recent decades, there were quite a few incidents involving pipelines/riser. On November 16, 1994, Hobbit Pipeline leak at Ship Shoal Block 281 was caused by bending failure of the pipe, which resulted in a 4,535 barrel oil spill. ${ }^{1}$ On 23 June 2007, a pipeline leakage on Main Pass Area Block 288 was caused by four corrosion holes in one pipe section. ${ }^{2}$ On April 20, 2010 the Deepwater Horizon, a semi-submersible mobile offshore drilling unit located in the Mississippi Canyon, experienced a blowout. Deepwater Horizon sank on April 22, 2010 and the riser collapsed with the platform. 11 workers died in the explosion. An oil leak ensued from the drill site which leaked 4.9 million barrels of oil into the Gulf of Mexico, making it the largest oil spill recorded in U.S. history (largest of its kind in the world history).

To prevent this kind of disaster, real-time structural health monitoring systems for risers in offshore deepwater platforms are needed to quickly determine whether there is damage and to activate contingency plans or mechanisms to initiate damage protection measures or to prevent complete blowout. 


\subsection{Current Riser Monitoring and Inspection Methods}

Risers are thoroughly inspected for damages before installation. Any periodic inspection or damage detection of the riser has to be done on-site and should not induce any damage to the riser. Hence, various non-destructive evaluation (NDE) methods have been introduced to the field of riser inspection. ${ }^{3}$ Typically, we can classify them into the following categories: Visual Inspection, Ultrasonic Technique (UT), Radiography, Electro-magnetic methods, Vibration based NDE and other techniques. The effectiveness of these NDE methods for detecting damage in deep water risers has not been evaluated or demonstrated.

Traditionally, visual inspection and manual ultrasonic testing for damage assessment are the main approaches for onshore inspection. However, visual inspection can only detect the external damage, cannot quantify damage and is too subjective. For deepwater risers, it is also impossible for the diver to get close to inspect the riser in ultra-deep sea. Hence, additional remotely operated vehicle is needed. For short-range $\mathrm{UT}^{4}$, its applicability in deepwater risers is excluded by its requirement of clean and smooth surface of the pipe and couplant to ensure the attachment and transmissibility between sensor and pipe surface. Long-range $\mathrm{UT}^{5}$ can only transmit around one wavelength of the guided wave into the riser, which results in neglecting the internal defects like corrosion if the wall of the riser is thicker than the wavelength. Safety issues and deepwater conditions keep radiography away from deepwater risers. According to Lozev et al., ${ }^{3}$ other new techniques like infrared thermography ${ }^{6}$ and acoustic emission ${ }^{7}$ technique are not applicable at deepwater scenarios.

In addition, all the electromagnetic testing methods currently in practice take advantage of the metallic characteristics of risers for damage detection. Magnetic flux leakage (MFL) is one of the most commonly used and the oldest in-line inspection methods to detect metal loss due to corrosion or gouging. Damages like weld defects or inclusions can be detected by MFL as well. With its ability to work efficiently using a permanent magnet, MFL can be a possible solution as local inspection method for deepwater risers.

Finally, Vibration-based methods have been widely used in civil structures, space structures, planes and cars. The advantage of vibration-based monitoring methods lies in the inherent simplicity, i.e., damage detection is based on the measurement of vibration alone. Unlike earlier methods which impose certain requirements on the surface to be monitored (UT), or techniques where the detection depth is limited by their input guided wave wavelength, vibration based techniques suffer from no such limitations. Further, unlike local inspection methods (like MFL) the results of the vibration based monitoring help us understand the global behaviour of the riser. Hence, vibration-based methods are a possible solution for deepwater riser monitoring systems.

Vandiver ${ }^{8}$ proposed to use the natural frequency change as an indicator of damage from the vibration response of fixed offshore platforms-where riser motion 
is not as important as the motion of platform tower. Similarly, a modal distribution method is proposed by Sweetman et al. ${ }^{9}$, which uses the significant change between the power spectrum from measured structural responses to indicate a change (such as damage) in primary system. This is also a frequency domain method based on output data, which is not applicable to deepwater risers with varying natural frequencies. Riveros et al. ${ }^{10}$ suggested that a statistical pattern recognition method with a combined model with auto-regressive (AR) and auto-regressive with exogenous inputs (ARX) works better than modal distribution method. However, in deepwater situation, the inputs are unknown, which means the method is not suitable. In addition, natural frequencies of the riser are time-varying due to the tension and touchdown points variation. Hence, there is a need for development of more reliable damage detection methods for deepwater risers that are based on vibration response.

\section{PROPOSED SHM SYSTEM FOR DEEPWATER RISERS}

\subsection{Strategy of the proposed SHM system}

As mentioned earlier, the safety of deepwater risers is essential for sustainable operation of offshore platforms. The structural health monitoring (SHM) system for deepwater risers is important to detect damage and perform repairs before failure occurs. ${ }^{11}$ Since combining global and local monitoring can greatly increase the accuracy of damage detection and fatigue estimation, a structural health monitoring system is proposed with local inspection using robotic Magnetic Flux leakage (MFL) sensors, which is efficient and provides high resolution estimate of wall thickness changes due to corrosion or damage, while proposed vibration-based system identification can estimate global damage locations and fatigue life.

The flowchart for the proposed strategy is shown in Figure 2. In the figure, global monitoring refers to continuous monitoring of the dynamic properties of risers in real-time. Vibration measurement can be accelerations, displacements and strains etc. The measured data is then processed to obtain the dynamic properties. The damage indexes (or fatigue estimation) are then derived from the dynamic properties. The damages, which are defined as dynamic property changes between intact and damage systems, can be located; damage severity can be estimated based on the magnitude of damage indices as well. If damage occurs according to the global monitoring system, a warning signal with damage information is sent to the control center. Then a robotic crawler carrying MFL sensors is sent to the predicted damage location to verify the warning and estimate its severity. Finally if both local and global monitoring indicates the occurrence of damage, the estimated service life and damage information is made available to the operators regarding the decision on repairing or replacement. 


\section{Vibration and Magnetic Flux Leakage(MFL)based \\ Deepwater Risers Monitoring System}

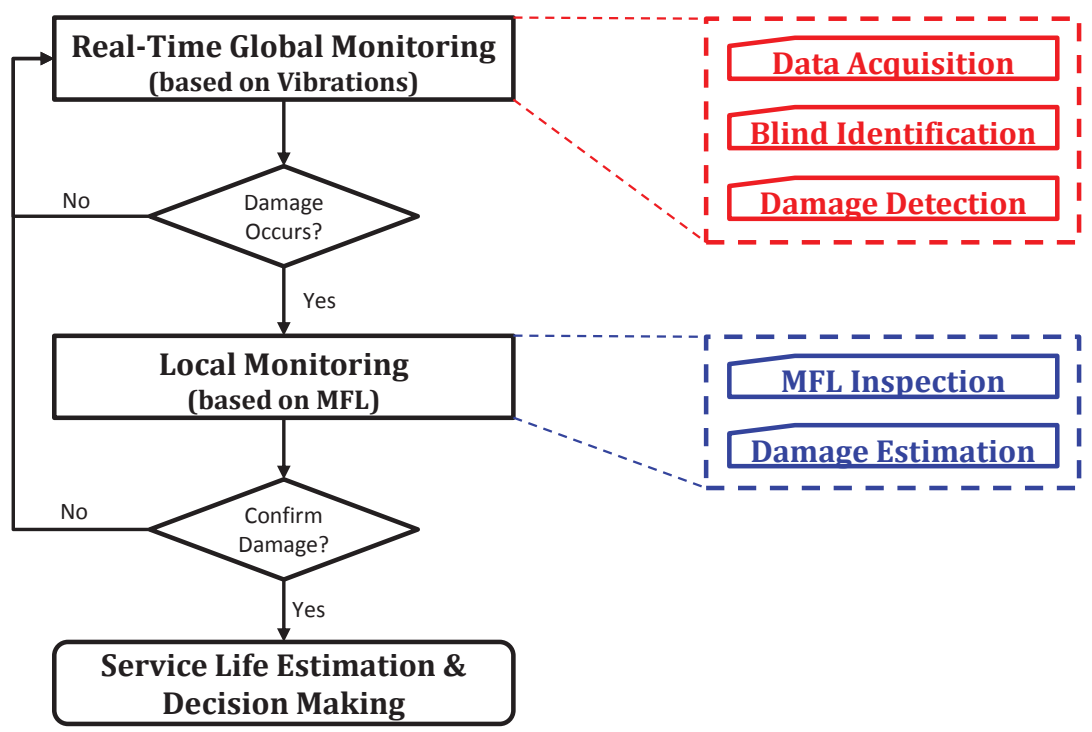

Fig. 2. Proposed Strategy for Deepwater SHM System

\subsection{Global Monitoring - based on Measured Riser Vibrations}

In this study, global monitoring is based on the vibration of deepwater risers. As shown in Figure 2, global monitoring is divided into three steps: Data Acquisition, Blind Identification and damage detection.

\subsubsection{Data Acquisition}

The response of risers is induced by vessel motions, wave and current etc., among which vortex-induce vibration (VIV), a special phenomenon caused by current passing by the riser, contains the most information about dynamic properties. When a bluff body is subjected to fluid flow with sufficiently large velocity, shedding vortices (a von Karman vortex street) will occur and exert oscillatory forces in both inline direction and the cross-flow direction. When one or more modes of bluff body are excited by the vortex shedding, large amplitude vibrations occur in steady state; the phenomenon is called vortex-induced vibration (VIV). The modal estimation is based on VIV. 


\subsubsection{Blind Identification and Damage Detection}

According to the definition of damage, both responses from undamaged and damaged system are needed to derive the changes of dynamic properties and their derivatives. To get the correct dynamic properties, a new modal identification and damage detection techniques for deepwater risers subjected to VIV is developed. Identification of frequency and mode shapes (dynamic properties) of deepwater risers from VIV responses using output only signal processing techniques is proposed based on wavelet ${ }^{12}$ and second order blind identification (SOBI) ${ }^{13}$ methods. SOBI is one of popular blind source separation method, based on the second order statistics independency. SOBI uses joint approximate diagonalization (JAD) to extract the independent source information form covariance matrices.

To get the damage location and severity, a new damage index derived from dynamic properties obtained from blind identification. The difference in the new

damage index between the undamaged and damaged cases is used to locate and quantify damage.

\section{PROPOSED OUTPUT ONLY MODAL EXTRACTION \& DAMAGE INDEX}

\subsection{Output Only Modal Identification - Wavelet Modified Second Order Blind Identification}

The complexity of the currents and measurement of hydrodynamic forces applied on risers makes it very difficult to measure the input excitation for deepwater risers. Hence, the riser damage detection becomes an output only problem, where outputonly modal estimation methods are needed.

A number of output-only modal identification methods such as frequency domain decomposition (FDD), Ibrahim Time Domain (ITD), eigensystem realization algorithms (ERA) and empirical mode decomposition (EMD), etc. have been developed for traditional structures. However, for deepwater risers, these methods may not be suitable because of frequency domain limitation, complex vibration modes, and low signal-to-noise ratio measurement. ${ }^{14}$

As a result, to overcome the above mentioned drawbacks, a time-frequency domain output only modal identification approach is proposed based on blind source separation (BSS).

\subsubsection{Blind Source Separation}

Blind Source Separation (BSS) is a statistical signal processing technique, which attempts to recover the individual unknown but statistically independent source components from mixed signals (measurements). The mixing process is shown as equation 3.1, where vector $X$ represents the mixed signals, matrix $A$ is the linear 
mixing matrix and vector $s$ represents the independent source components.

$$
\mathbf{X}=\mathbf{A s}
$$

The original problem for BSS can be referred to as the 'cocktail-party problem' defined by Cherry in the $1953^{15}$ about the analogy of human ears in differentiating what another person is speaking when quite a few people are speaking at the same time. Independent component analysis (ICA) is the most widely used BSS technique. ICA uses one of the simplest definitions of the measure of non-gaussianity, kurtosis, which is the fourth order cumulant of an independent source signal. For non-gaussian random variables, the kurtosis should be zero. However, due to the assumption of non-gaussian sources and higher statistical independency requirements, the application of ICA to civil engineering is limited to lightly damped structure with very good measurement system (i.e. high quality signals to get kurtosis), where damping is the key factor influencing the identification results.

To address the limitations of ICA, principle component analysis (PCA) and second order blind identification (SOBI) algorithms are developed based on the the second order statistical independency. PCA is also called as singular value decomposition (SVD) in mathematics, which provides the linearly uncorrelated sources from the mixed measurement. This orthogonality requirement of PCA limits its application in civil engineering where the mode shapes are not necessarily directly orthogonal to each other (but mass orthogonal). The inability to differentiate the repeated eigenvalues, sensitivity to measured noise and damping all limit PCA's application range ${ }^{13}$. Utilizing the JAD process, SOBI can overcome the limitations of PCA and works well in simulation studies as all the modes can be excited numerically. However, for a large scale civil structure like long-span bridges, the higher modes of vibration are not easily excited. In addition, spatially un-correlated noise strongly influences the identified results. The measurement noise need separate treatment before introducing covariance function. Last but not the least, traditional SOBI can only identify as many sources as the number of sensors, which is also known as under-determinate problem. Hence, a new BSS method is needed for civil engineering applications, especially for deepwater SHM system.

\subsubsection{Wavelet Modified Second Order Blind Identification Method}

To overcome the limitation of existing methods on the moderate damping condition, low signal-to-noise ratio measurement and limited number of sensors, SOBI is chosen to combine with wavelet transform method to recover the individual unknown but statistically independent source components from mixed signals (measurements) in time-frequency domain. The wavelet modified SOBI (WMSOBI) ${ }^{16}$ can be divided into four steps instead of three steps. Details are as follows (see Figure 3):

- Pre-Processing: Including centering, de-trending or filtering to remove the offset and slope in the measured signals; 


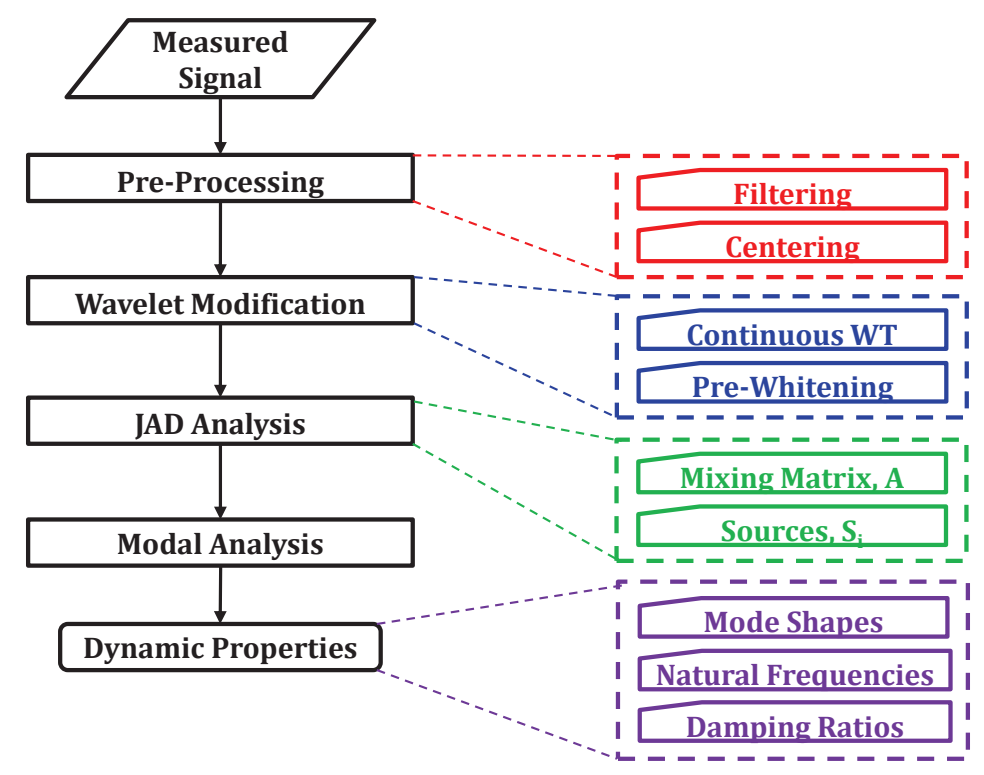

Fig. 3. Flowchart for WMSOBI

- Wavelet modification: Apply continuous wavelet transform (CWT) to preprocessed signal, results in the wavelet coefficients, $g_{k i}^{j}$, in the time-frequency domain using the following equations for a chosen scale, $\mathrm{j}$.

$$
\begin{array}{r}
x_{i}^{j}(t)=\sum_{k} g_{k i}^{j} \psi_{k}^{j}(t) \quad i=1,2, \cdots, N \\
s_{i}^{j}(t)=\sum_{k} f_{k i}^{j} \psi_{k}^{j}(t) \quad i=1,2, \cdots, N \\
\mathbf{X}=\mathbf{A} \mathbf{s} \Rightarrow g^{m}=\mathbf{A} f^{m}
\end{array}
$$

where $\psi(t)$ is the chosen wavelet, and $g_{k i}^{j}$ is the wavelet coefficients for scale $j$, sensor index $i$ and the time shift index $k ; s_{i}^{j}(t)$ is the $i$ th source signal for scale $j$, and $f_{k i}^{j}$ is the wavelet coefficients for scale $j$, sensor index $i$ and the time shift index $k$.

Doing CWT on both sides of equation 3.1 will result in equation 3.4, which proves the key idea of switching the time-domain measured signal with time-frequency domain wavelet coefficients. Taking a certain scale of wavelet coefficients which contains important information about sources as the input signal for joint approximate diagonalization (JAD). Usually, the dominant scale of wavelet coefficients is the first choice. And then either iteration to the second dominant wavelet coefficients or manually choosing based on which higher mode (scale) need to be 
identified. Then whitening using eigenvalue decomposition of correlation function $R_{g m}(0)$ to get the pre-whitened signal, $Y(t)$. It is the same procedure as SOBI.

- JAD Analysis: Apply Joint approximate diagonalization technique to the covariance matrices from the whitened wavelet coefficients, $Y(t)$, to get the joint diagonalizer, $\Psi(t)$. Then derive the de-mixing, $\mathbf{W}$, and mixing matrix, $\mathbf{A}$.

- Modal Analysis: Based on the de-mixing matrix, separate the measured signal to get the independent sources. If it is free vibration data, directly apply fast Fourier transform (FFT) and exponential decay method to get the damped natural frequency and damping ratio. If it is ambient excited response, approximate free vibration sources are obtained by de-mixing the cross-correlation functions of measured signal.

\subsection{Distributed Force Change (DFC) Index}

To overcome the limitation of existing damage index methods, a new damage index is proposed by choosing the fourth derivative of displacement mode shape, which is the second spatial derivative of the strain mode shape and proportional to the distributed force. Strain is proportional to curvature, which is the second spatial derivative of the displacement.

$$
\kappa_{y, z}=\frac{d^{2} y}{d z^{2}}=\frac{M_{y, z}}{E I}
$$

where $y$ is the displacement in $\mathrm{Y}$ direction, $z$ is the depth coordinate, $\kappa$ is the curvature in $\mathrm{Y}$ direction at depth $z$. EI is bending stiffness, while $M_{y, z}$ is the moment in $Y$ direction at the section at riser depth $z$.

The fourth derivative of displacement over the depth, which is proportional to the distributed force term, is as follows

$$
D F_{y, z}=\frac{d^{4} y}{d z^{4}}=-\frac{q_{y, z}}{E I}
$$

where $y$ is the displacement in $\mathrm{Y}$ direction, $z$ is the depth coordinate. $D F_{y, z}$ is proportional to the distributed force term, represented by $q_{y, z}$. The actual distributed force index is obtained using central difference method to process the identified curvature mode shape.

The possible false indication can be removed from distributed force change by multiplying it by a weighting function, which results in the weighted distributed force term for damage detection.

$$
W D F_{y, z}=D F_{y, z} \times \kappa_{y, z}^{2}
$$

where $\kappa_{y, z}$ represents the curvature/strain mode shape obtained from responses of undamaged structure for both undamaged and damaged cases.

The difference (change) between the undamaged and damaged WDF is defined as distributed force change (DFC) as an indicator for damages in the test structure.

To further take the advantage of multiple-modal participation, a combined DFC (CDFC) index is proposed by summing the DFCs of three dominant modes with 
consideration of signs of strain mode shape. The CDFC is obtained using the following equation:

$$
C D F C_{z}=\sum_{i=1}^{3}\left[\operatorname{sign}\left(\mathbf{C}_{\mathbf{z}}\right) * D F C_{z}\right]
$$

where $C_{z}$ represents normalized curvature (strain) mode shape values at location $z ; D F C_{z}$ represents the distributed force change values at location $z$.

\section{EXPERIMENTAL VERIFICATION}

\subsection{Experimental Setup}

To verify the proposed the SHM system, shown in Figure 4, a 186 inch long pipe with 2.5 inch inner diameter is placed horizontally and fixed at one end. Two shakers are attached to the pipe at 88.8 inch and 163.5 inch. The pipe is held using vertical cables and allowed to vibrate in the horizontal plane only. Eleven sensors (including strain gages and accelerometers) are installed in the horizontal plane along the pipe. The parameters of the test pipe are summarized in Table 1.

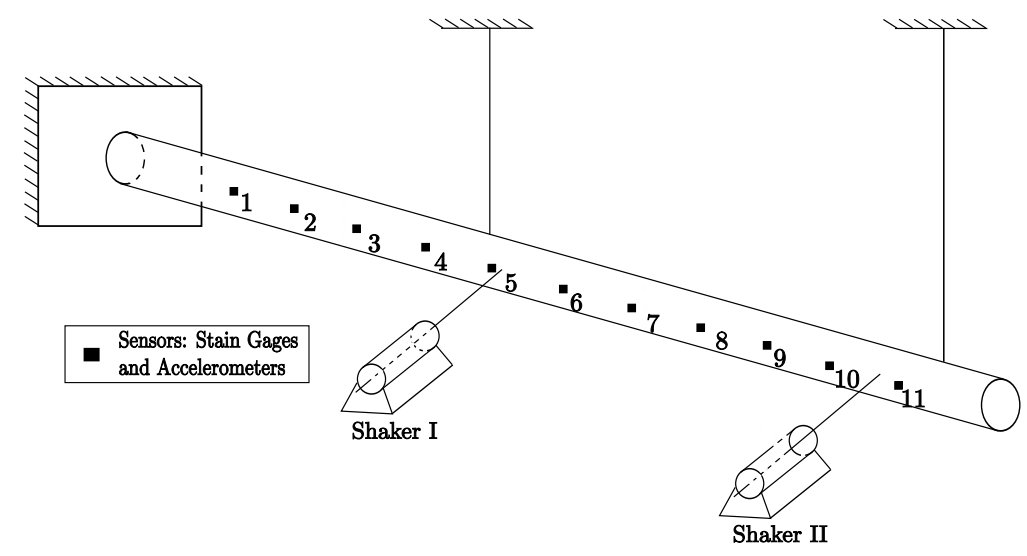

Fig. 4. Schematic of Experimental Setup and Sensor Locations

Table 1. Parameters of Test Pipe.

\begin{tabular}{cc|cc}
\hline Inner Diameter & $0.06375 \mathrm{~m}$ & Outer Diameter & $0.07334 \mathrm{~m}$ \\
\hline Density, $\rho$ & $7.85 \times 10^{3} \mathrm{~kg} / \mathrm{m}^{3}$ & Modulus of Elasticity, $E$ & $2 \times 10^{11} \mathrm{~N} / \mathrm{m}^{2}$ \\
\hline Area & $0.01 \mathrm{~m}^{2}$ & Length, $L$ & $4.7244 \mathrm{~m}$ \\
\hline Moment of Inertia, $I$ & $6.094 \times 10^{-7} \mathrm{~m}^{4}$ & Number of Elements, $N_{E}$ & 100 \\
\hline
\end{tabular}




\subsection{Experimental Procedure and Crack Simulation}

Multiple experiments are carried out with different structural health conditions and excitation cases. There are four different structural health conditions: intact structure, structure with single level I Crack, structure with single level II crack and structure with multiple cracks. A physical crack is located at $40 \%$ of the pipe length from the fixed end. The crack location is chosen not to overlap with the zero nodes in the first three vibration mode shapes. To study the damage severity effect, level I crack and level II crack are chosen at the same location with increasing damage. The second crack is created at $0.63 \mathrm{~L}$ from the fixed end. Detail information of the cracks is shown in Table 2.

Furthermore, three different excitation cases are tested for each structural health condition: Impact, White Noise and Chirp Signal.

Table 2. Dimension Information about Cracks

\begin{tabular}{ccccc}
\hline Name & Location & Width & Length & Depth \\
\hline Crack I Level I & $0.4 L=1.89 \mathrm{~m}$ & $2.32 \mathrm{~mm}$ & $26.67 \mathrm{~mm}$ & $2.54 \mathrm{~mm}$ \\
Crack I Level II & $0.4 L=1.89 \mathrm{~m}$ & $2.41 \mathrm{~mm}$ & $35.50 \mathrm{~mm}$ & $6.01 \mathrm{~mm}$ \\
Crack II & $0.63 L=3.01 \mathrm{~m}$ & $1.85 \mathrm{~mm}$ & $31.99 \mathrm{~mm}$ & $5.26 \mathrm{~mm}$ \\
\hline
\end{tabular}

\subsection{WMSOBI Verification using Intact Case}

The intact case test is used to validate the WMSOBI method by comparing with the modal properties identified using frequency response functions (FRFs) since input excitations are available. The identified modal frequencies from FRFs and WMSOBI are listed in Table 3. The results from FEM model are a little bigger in lower modes and a little smaller in higher mode, while the results from WMSOBI and FRFs are compatible.

Table 3. Identified Modal Properties

\begin{tabular}{cccc}
\hline Name & FEM & FRFs & WMSOBI \\
\hline Mode I & $2.757 \mathrm{~Hz}$ & $2.32 \mathrm{~Hz}$ & $2.55 \mathrm{~Hz}$ \\
Mode II & $17.31 \mathrm{~Hz}$ & $16.07 \mathrm{~Hz}$ & $16.59 \mathrm{~Hz}$ \\
Mode III & $48.46 \mathrm{~Hz}$ & $48.61 \mathrm{~Hz}$ & $49.50 \mathrm{~Hz}$ \\
\hline
\end{tabular}

In addition, the mode shapes for all three modes are plotted in Figure 5. The experimental values are scaled appropriately and the maximum normalized mode shapes from FEM. In Figure 5, solid lines represent the modes from FEM model (labeled as theoretical); line with stars represents the modes from WMSOBI and the line with triangles represents the modes from FRFs. Mode shapes from FEM model contain the values all along the pipe $(0 \mathrm{~m}$ to $4.74 \mathrm{~m})$, while the modes from experiments only locate at those measured locations, from $3 \mathrm{ft}$ to $13 \mathrm{ft}(0.9$ 
$\mathrm{m}$ to $3.9 \mathrm{~m}$ ). In Figure 5, it is evident that the mode shapes obtained from WMSOBI are compatible with those from FRFs. The comparable frequencies and mode
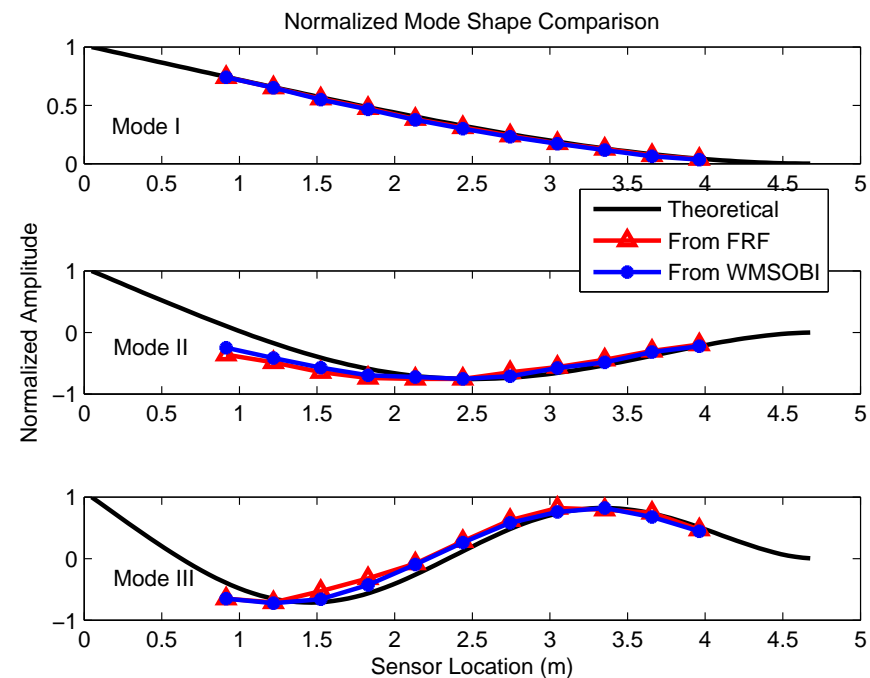

Fig. 5. Mode Shape Comparison

shapes identified from FRFs and WMSOBI demonstrate that the proposed WMSOBI method can identify reliable modal properties from output from measured response (output) only: the excitation signal is not considered in the WMSOBI.

\subsection{Damage Detection using Distributed Force Change (DFC) Index}

The mode shapes obtained using WMSOBI methods are used to evaluate the proposed damage detection method-weighted distributed force change (DFC) index. The proposed damage detection method is based on DFC index. The DFCs for each mode is obtained using central difference method according to equation 3.7.

\subsubsection{Damage Detection using DFC for Single Mode Shape}

The distributed force mode vectors and their corresponding differences are shown in Figure 6 for single crack, level I. In Figure 6, the black arrow points to where crack is introduced. For each individual mode comparison, DFC indices of mode II and mode III clearly indicate that there is damage close to sensor 4 and sensor 5. The DFC index close to the damage location has a typical shape of two small positive peaks on each side of one large negative peak. 

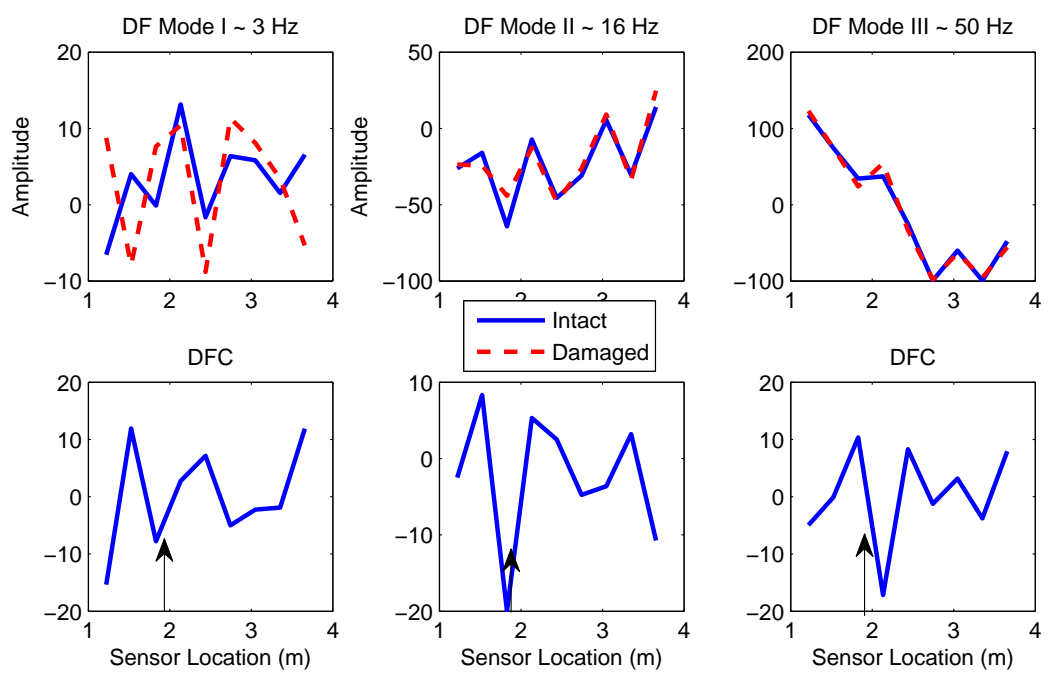

Fig. 6. DFC Damage Index - Single Crack Level I based on Mode I-III

The strain mode shapes obtained from experiments are proportional to curvature mode shape. According to Pandey ${ }^{17}$, curvature mode shape change can be used as damage indicator. Hence, the curvature mode shape changes between intact case and damaged case with single crack level I are shown in Figure 7. Upper plots in Figure 7 show the normalized experimental strain (curvature) mode shapes, and the lower plots show the curvature changes.
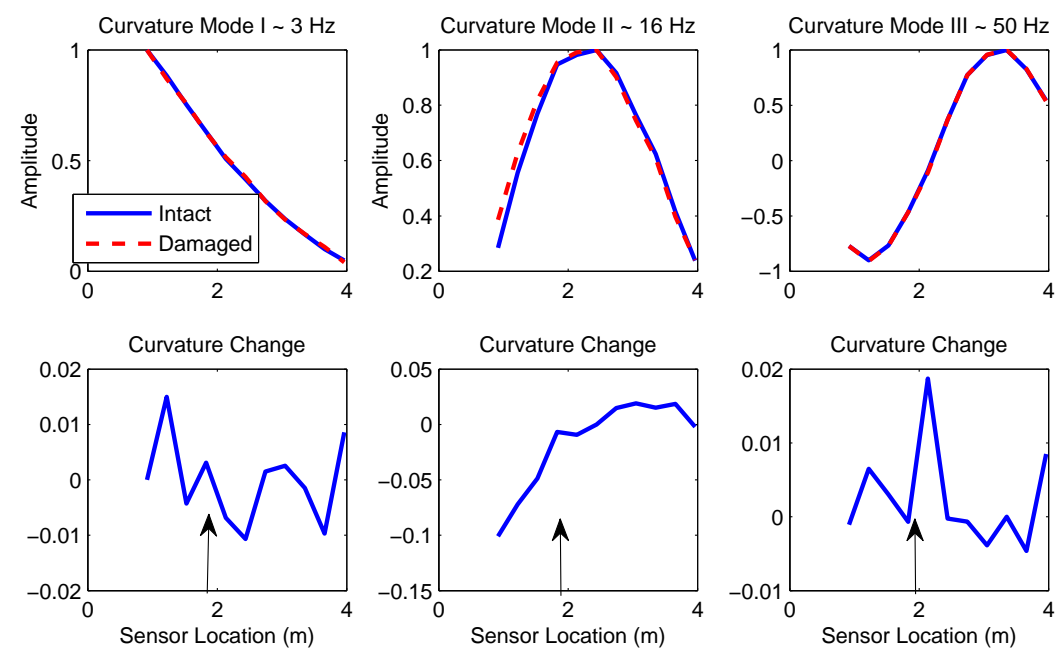

Fig. 7. Curvature Change for for Single Crack Level I based on Mode I-III 
Among all the three modes, only curvature change of mode III shows a clear peak around sensor 5 while the other modes do not provide a clear peak. In other words, curvature change based damage detection method cannot detect the damage location in this measurement setup. Further details on the curvature/DFC comparison can be found in Huang's thesis ${ }^{16}$.

Similar results for single crack with damage level II and multiple cracks, as shown in Figure 8 and Figure 9, validate the effectiveness of the proposed DFC index methods. The shape of inverse "W" shape of DFC index is the typical indicator where the damage occurs.
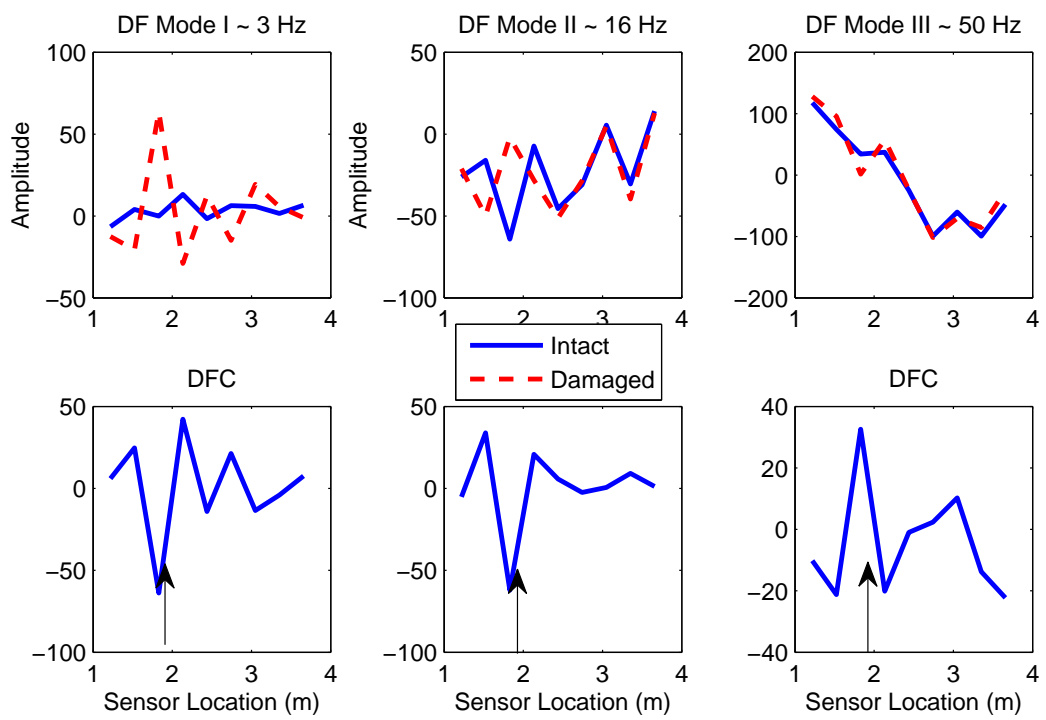

Fig. 8. DFC Damage Index - Single Crack Level II based on Mode I-III

When crack occurs, the corresponding bending stiffness reduction results in a small kink in curvature mode shape, which is proved by Pandey et al. ${ }^{17}$ using acceleration measurement. However, the strain gages measure the strains at the sensor locations. Since the curvature is proportional to strain measurement, the strain gage has to be within the damage affected area to detect the damage. On the other hand, the DFC index utilizes central difference method to get the second order derivatives in Equation 3.7. Utilizing three adjacent strain measurements in central difference process can cover a larger range than curvature change index. Hence, with the same strain measurements, the DFC index provides better identification results than curvature change index in this experiment. 

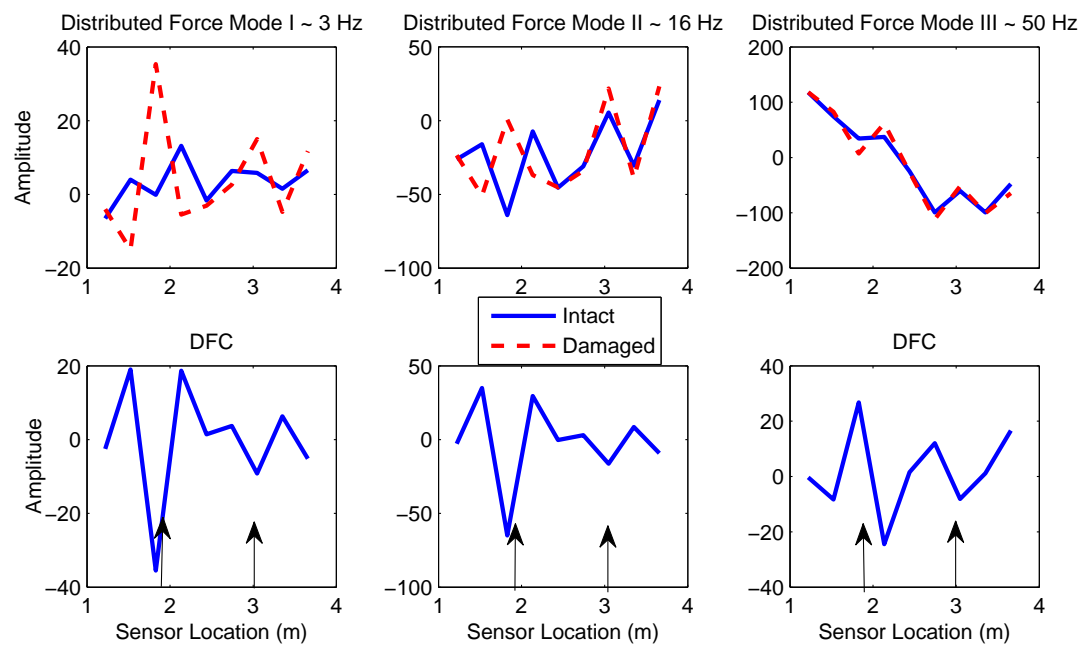

Fig. 9. DFC Damage Index - Multiple Cracks based on Mode I-III

\subsubsection{Damage Detection using CDFC for Multiple Mode Shapes}

To further take the advantage of multi-modal participation, the combined DFC (CDFC) index is calculated according to equation 3.8. The CDFC index for single crack (level I and II) and multiple cracks cases are shown in Figure 10.

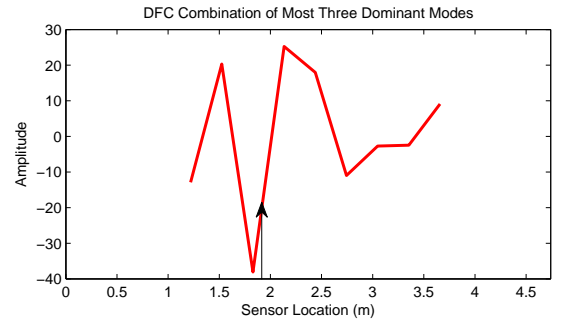

(a) Single Crack Level I

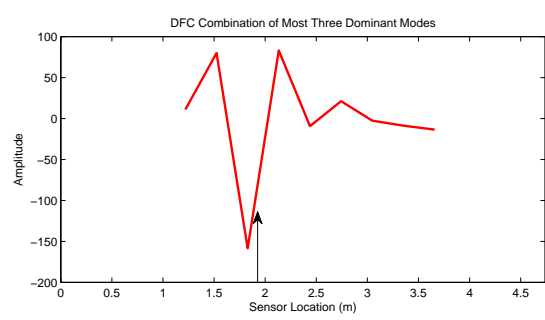

(b) Single Crack Level II

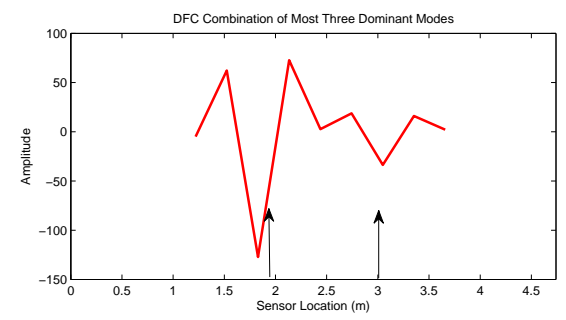

(c) Multiple Cracks

Fig. 10. CDFC Damage Index for Different Damage Cases 
The CDFC index for single crack with damage level I is shown in Figure 10(a). The typical damage shape clearly indicates that there is damage close to sensor while the other minor kinks in Figure 6 (mode I) do not have the same typical damage shape and have much smaller amplitudes, which means that there is no damage at those locations. Similarly, the CDFC shown in Figure 10(b) and (c) provide a clear and precise information about damage locations for both single and multiple crack cases.

Furthermore, the amplitude differences of CDFC peaks in Figure 10 are summarized in Table 4 with the corresponding cracks. The CDFC peak amplitudes in Table 4 and the crack severity shown in Table 2 show a very consistent correlation. The amplitude change of CDFC peaks for the same level II crack I could be a result of multiple crack interaction. Hence, the damage severity can be estimated using the peak amplitude of CDFC.

Table 4. Damage Severity and CDFC Index

\begin{tabular}{cccc}
\hline Single Crack Case & CDFC & Multi-Crack Case & CDFC \\
\hline Crack I Level I & -37 & Crack II & -32 \\
Crack I Level II & -150 & Crack I Level II & -125 \\
\hline
\end{tabular}

Note that the strain mode shapes are used in DFC calculation (Equation 3.7), therefore using CDFC to estimate the severity of crack should keep in mind of the influence of mode shapes.

\section{CONCLUSIONS}

A novel health monitoring strategy combining the global monitoring, based on vortex-induced vibration, and the local monitoring (inspection), based on magnetic flux leakage (MFL) phenomenon, provides a plausible solution for deepwater risers.

An experimental validation for the proposed SHM system was carried out. The experimental study validates the following conclusions:

- The Wavelet Modified Second Order Blind Identification (WMSOBI) method successfully estimates modal property and can provide as accurate results as modal analysis method using FRFs;

- Weighted Distributed Force Change (DFC) index serves as a reasonably good indicator of damage locations and severities, even for minor damages such as single crack with damage level I in the experiments. The negative peaks in the classic three-peak shape indicates the damage locations and the amplitude of the corresponding peaks indicate the severity;

- The combined DFC (CDFC) takes the advantage of multiple modes being excited at the same time and provides more reliable damage location and severity estimation. 


\section{Acknowledgments}

This research is supported by Department of Energy and Research Partnership to Secure Energy for America (RPSEA), Project No. 07121-DW1603D. This support is gratefully acknowledged.

\section{References}

1. Alvarado, A. and Bertges, W., Investigation of Shell Offshore Inc, Hobbit Pipeline Leak, Ship Shoal Block 281, November 16, 1994, U.S. Department of Interior, Mineral Management Services, Gulf of Mexico Regional Office: (New Orleans, 1994).

2. Gobert, A., Patton, F. and Williams, J., Investigation of Pipeline Leak Pipeline Segment No. 4582 Main Pass Area Block 288, 23 June 2007.,U.S. Department of Interior, Mineral Management Services, Gulf of Mexico Regional Office: (New Orleans, 2008).

3. Lozev, M., Smith, R. and Grimmett, B.m, Evaluation of methods for detecting andmonitoring of corrosion damage in risers, ASME Conference Proceedings, (2003) pp. 363-374.

4. Lozev, M. G., Smith, R. W. and Grimmett, B. B., Evaluation of methods for detecting and monitoring of corrosion damage in risers, Journal of Pressure Vessel Technology, vol. 127 , no. 3 , (2005) pp. 244-254.

5. Birring, A., Overview of factors affecting ultrasonic inspection of tension leg platforms, Proceedings of the Sixth ASME Symposium on Offshore Mechan-ics and Arctic Engineering (Houston, TX, 1984).

6. Thomas, R., Favro, L., Han, X. and Ouyang, Z., Infrared imaging of ultrasonically excited subsurface defects in materials, U.S. Patent-6236049 B1, (May 2001).

7. Yuyama, A. Fundamental aspects of acoustic emission applications to the problems caused by corrosion, in corrosion monitoring in industrial plants using nondestructive testing and electrochemical methods, ASTM STP 908 American Society of Testing and Materials, (Philadelphia. 1986) pp. 43-74.

8. Vandiver, J. K. Detection of structural failure on fixed platforms by measurement of dynamic response, Offshore Technology Conference, (1975), (Houston, TX).

9. Sweetman, B. and Choi, M. The modal distribution method: a new statistical algorithm for analyzing measured acceleration data, SPIE - Smart Structures and Materials 2006: Sensors and Smart Structures Technologies for Civil, Mechanical, and Aerospace Systems, (San Diego, CA, USA).

10. Riveros, C., Utsunomiya, M., Maeda, K., and Itoh, K. Vibration-based damage detection in flexible risers using time series analysis, Doboku Gakkai Ronbunshuu A, vol. 63, no. 3, (2007) pp. 423-433.

11. Huang, C., Structural health monitoring system for deepwater riser with vortexinduced vibration: nonlinear modeling, blind identification, fatigue/damage estimation and vibration control, Ph.D. Thesis, Rice University, (August 2012).

12. Nagarajaiah, S. and Varadarajan, N., Short time fourier transform algorithm for wind response control of buildings with variable stiffness TMD,Engineering Structures, vol. 27, no. 3, (2005) pp. 431-441.

13. Kerschen, G., Poncelet, F. and Golinval, J.-C., Physical interpretation of independent component analysis in structural dynamics, Mechanical Systems and Signal Processing, vol. 21, no. 4, (2007) pp. 1561-1575.

14. Nagarajaiah,S. and Basu,B. Output only modal identification and structural damage detection using time frequency \& wavelet techniques, Earthquake Engineering and Engineering Vibration, vol. 8, (2009) pp. 583C605. 
15. Cherry, E. C. Some experiments on the recognition of speech, with one and with two ears, ASA - The Journal of the Acoustical Society of America, vol. 25, no.5, (1953) pp. 975-979.

16. Huang, C., Nagarajaiah, S. and Varadarajan, N. Fatigue estimation in deepwater risers based on wavelets and second order blind identification, ASME 2012 31st International Conference on Ocean, Offshore and Arctic Engineering, (2012) pp. 829-838.

17. Pandey, A.K., Biswas, M. and Samman, M. M. Damage detection from changes in curvature mode shapes, Journal of Sound and Vibration, vol. 145, no.2, (1991) pp.321332 . 